Published in final edited form as:

Sleep Med. 2016 September ; 25: 156-161. doi:10.1016/j.sleep.2016.05.009.

\title{
Obstructive sleep apnea and incident type 2 diabetes
}

\author{
Mako Nagayoshia,b, ${ }^{,}$, Naresh M. Punjabic, Elizabeth Selvin ${ }^{d}$, James S. Pankow ${ }^{a}$, Eyal \\ Shahare, Hiroyasu Iso $^{f}$, Aaron R. Folsom $^{\mathrm{a}}$, and Pamela L. Lutsey ${ }^{\mathrm{a}}$ \\ aDivision of Epidemiology and Community Health, University of Minnesota, Minneapolis, MN, \\ USA
}

${ }^{b}$ Department of Community Medicine, Nagasaki University Graduate School of Biomedical Science, Nagasaki, Japan

'Division of Pulmonary and Critical Care Medicine, Johns Hopkins University School of Medicine, Baltimore, MD, USA

${ }^{\mathrm{d} D e p a r t m e n t}$ of Epidemiology and the Welch Center for Prevention, Epidemiology and Clinical Research, Johns Hopkins Bloomberg School of Public Health, Baltimore, MD, USA

eDepartment of Epidemiology and Biostatistics, Mel and Enid Zuckerman College of Public Health, University of Arizona, Tucson, AZ, USA

fDivision of Preventive and Environmental Medicine, Osaka University, Osaka, Japan

\section{Abstract}

Objectives-To determine whether severity of obstructive sleep apnea is associated with incident diabetes in middle-aged and older adults.

\begin{abstract}
Methods-A prospective analysis of 1,453 non-diabetic participants of both the Atherosclerosis Risk in Communities Study and the Sleep Heart Health Study (mean age 63 years, 46\% male) had in-home polysomnography (1996-1998) and were followed for incident diabetes. Using the apneahypopnea index derived from home polysomnography, study participants were categorized as follows: $<5.0$ (normal), 5.0-14.9 (mild), 15.0-29.9 (moderate), and 230.0 events/h (severe). Incident diabetes was ascertained during annual follow-up telephone calls through 2013.
\end{abstract}

Results-During a median follow-up of 13 years, there were 285 incident diabetes cases among the 1453 participants. Participants with severe obstructive sleep apnea were at greater risk of incident diabetes compared to persons classified as normal after adjustment for confounders

\footnotetext{
*Corresponding author. Department of Community Medicine, Nagasaki University Graduate School of Biomedical Science, 1-12-4 Sakamoto, Nagasaki 852-8523, Japan. Tel.: +81 95975 0121; fax: +81 95975 0122. mnagayoshi@ nagasaki-u.ac.jp (M. Nagayoshi). Publisher's Disclaimer: This is a PDF file of an unedited manuscript that has been accepted for publication. As a service to our customers we are providing this early version of the manuscript. The manuscript will undergo copyediting, typesetting, and review of the resulting proof before it is published in its final citable form. Please note that during the production process errors may be discovered which could affect the content, and all legal disclaimers that apply to the journal pertain.

Conflicts of Interest

This was not an industry supported study. All authors have no relevant conflict of interest to disclose. The ICMJE Uniform Disclosure Form for Potential Conflicts of Interest associated with this article can be viewed by clicking on the following link: http://dx.doi.org/ 10.1016/j.sleep.2016.05.009.

Supplementary data to this article can be found online at doi:10.1016/j.sleep.2016.05.009.
} 
including body mass index and waist circumference $(1.71[1.08,2.71])$. The association between severe obstructive sleep apnea and incident diabetes was similar when analyses were restricted to obese individuals.

Conclusions-Severe obstructive sleep apnea was associated with greater risk of incident diabetes, independent of adiposity in a community-based sample. Healthcare professionals should be cognizant of the high prevalence of OSA in the general population and the potential link to incident diabetes.

\section{Keywords}

Epidemiology; Obstructive sleep apnea; Diabetes; Obesity

\section{Introduction}

Given the morbidity and mortality associated with type 2 diabetes mellitus [1], the identification of potentially modifiable risk factors for diabetes remains a clinical and public health priority. Obstructive sleep apnea (OSA) is a common condition with approximately $13 \%$ of adult men and $6 \%$ of adult women having moderate to severe undiagnosed OSA [2]. Evidence collected over the last two decades from clinical and community-based studies suggests that OSA is associated with insulin resistance, glucose intolerance, and type 2 diabetes independent of the confounding effects of obesity [3,4]. Moreover, data from murine models [5] and experimental human studies [6] have also shown that exposure to intermittent hypoxia and sleep fragmentation can lead to alterations in insulin sensitivity and glucose disposal. Thus, observational [7-11] and experimental data[12,13] are convergent and implicate OSA as an independent risk factor for development of diabetes. Unfortunately, interventional data examining the effects of OSA treatment on metabolic outcomes such as insulin sensitivity, glucose tolerance, and glycosylated hemoglobin have been equivocal $[12,13]$, and thus have raised significant doubt regarding the role of OSA in the pathogenesis of type 2 diabetes. Thus, recommendations regarding case-identification for OSA in those at risk for metabolic dysfunction need to be tempered by the lack of a strong empirical base. Part of the challenge in interpreting the available interventional data on the effects of OSA treatment on metabolic parameters is due to the wide range of methodological limitations in the available studies [7]. Relatively limited sample sizes, poor adherence with treatment, and duration of treatment are some of the many pitfalls in the treatment-related data available to date. There is also a dearth of longitudinal data on whether untreated OSA in those free of diabetes increases the predisposition for developing diabetes [14,15]. Longitudinal evidence to support temporality of the association between OSA and diabetes, and whether the association is independent of obesity, is lacking. Availability of such evidence would motivate case-identification and early intervention for OSA to mitigate some of the risk for developing diabetes. The longitudinal studies that have examined the impact of OSA on incident diabetes have either been based on clinical samples [10,11] or have not included full montage polysomnography $[9,11]$. Therefore, using data from a subset of Atherosclerosis Risk in Communities Study (ARIC) participants who took part in the Sleep Heart Health Study (SHHS), the current study sought to determine whether OSA is associated with incident diabetes, independent of known confounding factors such as obesity. 


\section{Methods}

\subsection{Study Population}

In 1987-1989, using population-based probability sampling, the ARIC study enrolled 15,792 adults (aged 45-64 years) from four US communities [16]. A total of four follow-up visits have taken place (1990-1992, 1993-1995, 1996-1998, and 2011-2013) since the initial visit. During the fourth ARIC visit (1996-1998), which constitutes baseline for the present study, a subset of 1920 ARIC participants from the study sites of Washington County, MD and suburban Minneapolis, MN were recruited into the SHHS [17] and underwent an in-home overnight polysomnogram. For the current analysis, 467 participants were excluded for the following reasons: (1) prevalent diabetes at baseline defined as a fasting glucose $\geq 126 \mathrm{mg} / \mathrm{dL}$ or non-fasting glucose of $\geq 200 \mathrm{mg} / \mathrm{dL}$, self-reported history of physician-diagnosed diabetes, or medication use for diabetes over the last two weeks ( $N=261)$; (2) non-white race ( $N=11)$; (3) lack of follow-up information $(N=28)$; and (4) incomplete data on OSA severity $(N=167)$. The final analytic sample included 1453 participants (Fig. 1). Institutional review boards at each participating institution approved the study and all participants provided written informed consent.

\subsection{Sleep Assessments}

An overnight unattended in-home polysomnogram was conducted using a portable monitor (PS-2 System; Compumedics Limited, Abbotsford, Victoria, Australia), using methods previously described [18]. As in previous analyses of SHHS data, an apnea was defined if there was an absence or near absence of airflow (at least $<25 \%$ of baseline) for at least $10 \mathrm{~s}$ [18]. Hypopnea was defined as a 30\% decrease in the amplitude of the airflow for at least 10 s. The apnea-hypopnea index (AHI) was derived as the number of obstructive apneas (regardless of the oxygen desaturation level) plus hypopneas (with at least a $4 \%$ decrease in oxygen saturation) per hour of sleep, which corresponds to current definitions used by Medicare for reimbursement. Central sleep-disordered breathing events were excluded from the AHI definition. Participants were categorized based on the AHI as follows: $<5.0$ events/h (normal), 5.0-14.9 events/h (mild), 15.0-29.9 events/h (moderate), and $\geq 30.0$ events/h (severe).

Nocturnal hypoxemia was characterized by the average oxyhemoglobin saturation during non-rapid eye movement (non-REM) and REM sleep, and by the oxygen desaturation index which was defined as the average number of $4 \%$ or greater oxygen desaturation events per hour of sleep (4\% ODI). Arousals were identified as abrupt shifts $\geq 3 \mathrm{sec}$ in electroencephalogram frequency [19]. The arousal index was defined as the average number of arousals per hour of sleep. As detailed in the results, only about 5\% of the ARIC sample was classified as having severe OSA, defined by AHI $\geq 30$ events/h. In order to make the distributions of nocturnal hypoxemia and the arousal index comparable to the definition of severe OSA, for analytic purposes we categorized these variables into quintiles, and then further divided the most adverse category at the 5 th percentile. Thus, for average oxyhemoglobin saturation, the main comparison is the lower 5th percentile of the distribution to those in the highest quintile (percentiles 80-100). For 4\% ODI and the 
arousal index the main comparison is of those in the $\geq 5$ th percentile to those in the lowest quintile (percentiles 0-20).

Habitual sleep duration per week was assessed with the following questions: "How much sleep do you usually get at night (or in your main sleep period): on weekdays or workdays?" and "on weekends or non-work days?" Average usual sleep time (h) per week was determined as a weighted average: [(habitual total sleep time during the workdays) $* 5+$ (habitual total sleep time during the weekends)*2]/7.

\subsection{Incident Diabetes and Assessment of Covariates}

Diabetes was ascertained using information collected from ARIC annual follow-up phone calls that took place from the baseline visit (1996-1998) through 2013. Participants who reported physician-diagnosed diabetes or diabetes medication use were categorized as having incident diabetes. Covariates and potential mediators were assessed at the baseline examination (1996-1998). Information on socioeconomic status, marital status, smoking status, and alcohol use was self-reported. Measures of socioeconomic status included educational attainment (less than high school, high school graduate, beyond high school), income ( $<\$ 25,000, \$ 25,000-\$ 49,999, \$ 50,000)$, and occupation (managerial and professional job or not). Physiologic variables were measured by trained technicians. BMI was assessed as weight $(\mathrm{kg})$ divided by height $(\mathrm{m})$ squared. Waist circumference was measured at the umbilicus. High-sensitivity C-reactive protein (hsCRP) was measured using a latex-particle enhanced immunoturbidimetric assay kit (Roche Diagnostics, Indianapolis, IN 46250, USA).

\subsection{Statistical analysis}

Descriptive statistics were generated for all sleep-related variables and covariates, stratified by OSA severity. Analysis of variance and $\chi^{2}$ tests were used to examine differences in means for continuous variables or in proportions for categorical variables, respectively. Spearman correlation coefficients were calculated among the key variables of interest. Cox proportional hazards regression models were used to estimate hazard ratios (HRs) and 95\% confidence intervals (CIs) relating OSA severity to incident diabetes. The linear trend was tested by including the OSA severity categories in the models as a linear term. Follow-up time was calculated from the date of the baseline visit until the date of the telephone call during which incident diabetes was first reported, the date of last contact if the participant was lost to follow-up, or the date of the 2013 annual follow-up phone call, whichever came first.

We constructed and compared a series of nested models: Model 1 included age, sex, and center as covariates. Model 2 additionally adjusted for socioeconomic status (educational attainment, income, occupation), marital status, and behavioral risk factors (smoking status, alcohol use, physical activity). Model 3 further adjusted for adiposity (BMI and waist circumference). The proportional hazards assumption was evaluated by testing the interaction between OSA categories and the natural log of follow-up time, and by visual inspection of graphs of the survival function vs survival time, stratified by OSA categories. In secondary analyses we assessed whether systemic inflammation mediated the association 
between OSA and incident diabetes by adding hsCRP to covariates in Model 3. To assess whether self-reported sleep duration confounded the association between OSA and incident diabetes, habitual sleep duration was added to the covariates in Model 3. As both OSA and diabetes are strongly influenced by obesity, we conducted analyses that were restricted to participants with a BMI $\geq 30 \mathrm{~kg} / \mathrm{m}^{2}$. Additionally, effect modification of the association between OSA and diabetes by sex was assessed by including cross-product terms of the sex and OSA variables in Model 3. Finally, sensitivity analyses were conducted in which participants who at baseline did not self-report being diabetic, but who had high blood glucose (fasting glucose $\geq 126 \mathrm{mg} / \mathrm{dL}$ or non-fasting glucose of $\geq 200 \mathrm{mg} / \mathrm{dL}$ ), were retained in the analysis. In secondary analyses, we explored the associations of other indices of sleep (ie, hemoglobin desaturation during REM and non-REM sleep, 4\% ODI, and the arousal index) with risk of incident diabetes. These exposures were modeled categorically. All analyses were performed using SAS 9.3 (SAS Institute Inc., Cary, NC, USA).

\section{Results}

The 1453 participants in the final analytic sample were 53.5\% female, on average 62.5 years old, and had a mean BMI of $28.3 \mathrm{~kg} / \mathrm{m}^{2}$. Over a median of 12.8 years ( $\max =13.7$ years) of follow-up, 285 incident diabetes cases occurred, yielding a crude total diabetes incidence of 17.6 per 1000 person-years. Of the sample, $5.7 \%$ had severe OSA, $12.9 \%$ had moderate OSA, 28.8\% had mild OSA, and 52.6\% were without OSA (Table 1). Those with severe OSA were more likely to be older, male, current or former smokers, hypertensive, and had a higher BMI and waist circumference than those without OSA. Moreover, compared to the participants without OSA, those with severe OSA reported shorter habitual sleep duration and had a lower average oxyhemoglobin saturation, higher 4\% ODI, and arousal index (Table 2). AHI was positively correlated with BMI $(r=0.39)$ and waist circumference $(r=$ $0.44)$ and inversely correlated with average oxyhemoglobin saturation during REM sleep $(r$ $=-0.52)$ and non-REM sleep $(\mathrm{r}=-0.44)$. Furthermore, the correlation of AHI and average oxyhemoglobin saturation during REM sleep and non-REM sleep was stronger in obese than in non-obese participants $(r=-0.56$ and $r=-0.44$ in obese, and $r=-0.44$ and $r=-0.36$ in non-obese, respectively).

A dose-response association was observed between OSA severity and incident diabetes across all statistical models ( $p$-trend $\leq 0.01$ ) (Table 3). After adjustment for demographics and health behaviors, participants with severe OSA were at 2.75-times (95\% CI: 1.81, 4.19) greater risk of incident diabetes than participants without OSA. The association remained statistically significant despite adjustment for adiposity (HR: 1.71 [1.08, 2.71]). With additional adjustment for hsCRP, results were slightly attenuated (HR: 1.57 [0.98, 2.54]). There was no evidence that sex modified the association between OSA severity and incident diabetes. As the majority of participants with OSA were obese, additional analyses restricted to participants of BMI $230 \mathrm{~kg} / \mathrm{m}^{2}$ were conducted (Table 3). Even after adjusting for adiposity, obese participants with severe OSA were at 2.03-times $(1.20,3.44)$ greater risk of incident diabetes than obese participants without OSA. Moreover, sleep duration did not confound the association between OSA and incident diabetes in either the full sample or when restricted to obese participants. In sensitivity analyses, the association between OSA and incident diabetes was not appreciably changed when we retained participants who at 
baseline had elevated blood glucose level but did not report diagnosed diabetes $(N=64)$ (see Table E1 in the online data supplement).

Associations between several markers of nocturnal hypoxemia and sleep fragmentation namely average oxyhemoglobin saturation during REM and non-REM sleep, 4\% ODI, and the arousal index - and risk of incident diabetes in the full sample and among obese participants are provided in Tables E2 and E3 in the online data supplement, respectively. Overall, more adverse levels of nocturnal hypoxemia and sleep fragmentation were associated with greater risk of incident diabetes, though associations were at times attenuated with adjustment for adiposity (Table E2). When the sample was restricted to obese individuals, relative to those in the lowest categories, being in the highest categories of $4 \%$ ODI and the arousal index were associated with greater risk of incident diabetes, even after adjusting for adiposity (Table E3). However, in analyses restricted to obese individuals, hemoglobin desaturation was not significantly associated with diabetes risk.

\section{Discussion}

Severe OSA, as assessed by in-home polysomnography, was associated with a $71 \%$ increased risk of incident type 2 diabetes, independent of potential diabetes risk factors including BMI and waist circumference, in this community-based sample. The current study provides strong evidence for a longitudinal association between OSA and incident diabetes. The association persisted even after the analyses were restricted to obese individuals, providing further credence to the idea that OSA increases the risk for diabetes, and is not merely linked through a common correlation with adiposity. These findings support recommendations from the International Diabetes Federation consensus statement that "OSA patients should be routinely screened for markers of metabolic disturbance and cardiovascular risk", to, including markers of glucose homeostasis [20].

Our findings are consistent with results from several clinic-based studies [10,11], and a recently published historical cohort of patients undergoing diagnostic sleep studies, which found that those with severe OSA were at $30 \%$ greater risk of incident diabetes, independent of BMI [15]. Population-based studies that have assessed the prospective association have yielded mixed results partly because of the relatively small number of incident cases of diabetes in participants with moderate-severe OSA $[7,8]$. While the degree of nocturnal intermittent hypoxemia as assessed through pulse-oximetry has been associated with incident diabetes risk in a Japanese population-based sample [9], the lack of polysomnography in that study is a notable limitation. Longitudinal studies (eg, Nurses' Health Study) that have used self-reported sleep symptoms, such as habitual snoring, as surrogates for OSA have also found these symptoms to be associated with higher diabetes risk $[21,22]$. The current study extends these seminal observations by demonstrating that moderate to severe OSA as defined by overnight polysomnography does predict incident diabetes in a large community sample that has a relatively long period of follow-up. We also provided novel evidence that both nocturnal hypoxemia and sleep fragmentation are associated with OSA risk. 
Clinical trials on whether treatment of OSA with continuous positive airway pressure (CPAP) improves insulin resistance and glycemic control in non-diabetic and diabetic patients with OSA have yielded mixed results [12]. The lack of a consistent effect could be related to a number of methodological limitations including the lack of availability of an adequately powered randomized clinical trial $[4,20]$. It is important to recognize that the impact of OSA on development of diabetes may, in fact, be distinct from its effects on glycemic control. It is certainly possible that OSA is an independent risk factor for diabetes but has a less important role for glycemic control after the onset of diabetes. Alternatively, it is also plausible that with beta cell failure and subsequent development of diabetes, the potential effects of OSA on insulin secretion and sensitivity are irreversible [23]. There are several possible mechanisms underlying the association between OSA and incident diabetes. OSA-related intermittent hypoxemia and recurrent arousals may affect glucose metabolism through activation of the sympathetic nervous system [24], changes in activity of the hypothalamic-pituitary-adrenal axis [25] with increasing levels of circulating corticosteroids, formation of reactive oxygen species [26], and increases in inflammatory markers (eg, hsCRP, interleukin-6 and tumor necrosis factor-a) [27] and adipocyte-derived factors [28] (eg, leptin, adiponectin, and resistin).

The findings of the current study should be interpreted in light of several limitations. The assessment of OSA was conducted in participants' homes rather than at a sleep laboratory. However, AHI measured by in-home polysomnography is highly concordant with polysomnography in the laboratory [19]. Second, measurement error (and subsequent misclassification) in ascertainment of diabetes cases may have occurred as these data were self-reported. Previous work in the ARIC study has demonstrated that self-reported diabetes has high specificity (85-91\%) and moderate sensitivity (56-80\%) when compared with objective definitions [29]. It is also possible that participants with diagnosed OSA receive more frequent medical care and are thus more likely to have diabetes identified than those without diagnosed OSA. Measurement error may have also been a problem for some of the confounders, such as physical activity, which could result in residual confounding. Third, the analytic sample was limited to Caucasians and thus the results may not be generalizable to other races. Lastly, information on OSA treatment during the follow-up period was not available.

Despite these limitations, our study has several strengths. In-home polysomnography enabled us to objectively characterize OSA. In addition, the SHHS also assessed a variety of sleep characteristics which, when coupled with the wealth of information from the ARIC study, allowed a rigorous assessment as to whether the association between OSA and incident diabetes was independent of known diabetes risk factors including BMI and waist circumference. Lastly, the ARIC study identified incident diagnosed diabetes annually using a standardized follow-up call.

\section{Conclusion}

In summary, in a community-based longitudinal study, objectively measured severe OSA was associated with greater risk of incident diabetes. Importantly, the association was independent of BMI and waist circumference, and persisted even when the sample was 
restricted to obese participants. These findings complement the existing evidence suggesting a causal relationship between severe OSA and risk of diabetes, and support recommendations from the International Diabetes Federation that health professionals working in both type 2 diabetes and OSA should adopt clinical practices to ensure that patients presenting with one condition are considered for the other [20]. Furthermore, additional large and well-designed randomized clinical trials to examine whether treating severe OSA can lower glucose, and possibly contribute to diabetes prevention, may be warranted.

\title{
Supplementary Material
}

Refer to Web version on PubMed Central for supplementary material.

\section{Acknowledgments}

\author{
Funding
}

This study was supported by the National Heart, Lung, and Blood Institute contracts (HHSN268201100005C, HHSN268201100006C, HHSN268201100007C, HHSN268201100008C, HHSN268201100009C, HHSN268201100010C, HHSN268201100011C, and HHSN268201100012C), and the cooperative agreements U01HL53934 (University of Minnesota) and U01HL64360 (Johns Hopkins University). P.L. was supported by the National Heart, Lung and Blood Institute (R21 HL121412), E.S. by the National Institute of Diabetes and Digestive and Kidney Diseases (R01 DK089174), and N.M.P. by the National Heart, Lung and Blood Institute (R01 HL075078). Reagents for the high sensitivity C-reactive protein assays were donated by the Roche Diagnostics Corporation.

The authors thank the staff and participants of the SHHS-ARIC study for their important contributions.

\section{References}

1. Fox CS, Golden SH, Anderson C, Bray GA, Burke LE, de Boer IH, et al. Update on Prevention of Cardiovascular Disease in Adults With Type 2 Diabetes Mellitus in Light of Recent Evidence: A Scientific Statement From the American Heart Association and the American Diabetes Association. Circulation. 2015; 132:691-718. [PubMed: 26246173]

2. Peppard PE, Young T, Barnet JH, Palta M, Hagen EW, Hla KM. Increased Prevalence of SleepDisordered Breathing in Adults. American Journal of Epidemiology. 2013; 177:1006-1014. [PubMed: 23589584]

3. Moon K, Punjabi NM, Aurora RN. Obstructive Sleep Apnea and Type 2 Diabetes in Older Adults. Clinics in Geriatric Medicine. 2015; 31:139-147. [PubMed: 25453306]

4. Punjabi NM. Do sleep disorders and associated treatments impact glucose metabolism? Drugs. 2009; 69(Suppl 2):13-27. [PubMed: 20047348]

5. Polotsky VY, Li J, Punjabi NM, Rubin AE, Smith PL, Schwartz AR, et al. Intermittent hypoxia increases insulin resistance in genetically obese mice. The Journal of physiology. 2003; 552:253264. [PubMed: 12878760]

6. Louis M, Punjabi NM. Effects of acute intermittent hypoxia on glucose metabolism in awake healthy volunteers. Journal of Applied Physiology. 2009; 106:1538-1544. [PubMed: 19265062]

7. Reichmuth KJ, Austin D, Skatrud JB, Young T. Association of Sleep Apnea and Type II Diabetes. American Journal of Respiratory and Critical Care Medicine. 2005; 172:1590-1595. [PubMed: 16192452]

8. Marshall NS, Wong KKH, Phillips CL, Liu PY, Knuiman MW, Grunstein RR. Is Sleep Apnea an Independent Risk Factor for Prevalent and Incident Diabetes in the Busselton Health Study? Journal of Clinical Sleep Medicine : JCSM : Official Publication of the American Academy of Sleep Medicine. 2009; 5:15-20. [PubMed: 19317376] 
9. Muraki I, Tanigawa T, Yamagishi K, Sakurai S, Ohira T, Imano H, et al. Nocturnal intermittent hypoxia and the development of type 2 diabetes: the Circulatory Risk in Communities Study (CIRCS). Diabetologia. 2010; 53:481-488. [PubMed: 19946661]

10. Botros N, Concato J, Mohsenin V, Selim B, Doctor K, Yaggi HK. Obstructive Sleep Apnea as a Risk Factor for Type 2 Diabetes. The American Journal of Medicine. 2009; 122:1122-1127. [PubMed: 19958890]

11. Celen YT, Hedner J, Carlson J, Peker Y. Impact of gender on incident diabetes mellitus in obstructive sleep apnea: a 16-year follow-up. J Clin Sleep Med. 2010; 6:244-250. [PubMed: 20572417]

12. Schlatzer C, Schwarz EI, Kohler M. The effect of continuous positive airway pressure on metabolic variables in patients with obstructive sleep apnoea. Chronic Respiratory Disease. 2014; 11:41-52. [PubMed: 24431410]

13. Iftikhar IH, Khan MF, Das A, Magalang UJ. Meta-analysis: Continuous Positive Airway Pressure Improves Insulin Resistance in Patients with Sleep Apnea without Diabetes. Annals of the American Thoracic Society. 2013; 10:115-120. [PubMed: 23607839]

14. Wang X, Bi Y, Zhang Q, Pan F. Obstructive sleep apnoea and the risk of type 2 diabetes: a metaanalysis of prospective cohort studies. Respirology. 2013; 18:140-146. [PubMed: 22988888]

15. Kendzerska T, Gershon AS, Hawker G, Tomlinson G, Leung RS. Obstructive Sleep Apnea and Incident Diabetes. A Historical Cohort Study. American Journal of Respiratory and Critical Care Medicine. 2014; 190:218-225. [PubMed: 24897551]

16. The ARIC Investigators. The Atherosclerosis Risk in Communities (ARIC) Study: Design and objectives. American Journal of Epidemiology. 1989; 129:687-702. [PubMed: 2646917]

17. Quan SF, Howard BV, Iber C, Kiley JP, Nieto FJ, O'Connor GT, et al. The Sleep Heart Health Study: design, rationale, and methods. Sleep. 1997; 20:1077-1085. [PubMed: 9493915]

18. Redline S, Sanders MH, Lind BK, Quan SF, Iber C, Gottlieb DJ, et al. Methods for obtaining and analyzing unattended polysomnography data for a multicenter study. Sleep Heart Health Research Group. Sleep. 1998; 21:759-767. [PubMed: 11300121]

19. Redline S, Tosteson T, Boucher MA, Millman RP. MEasurement of sleep-related breathing disturbances in epidemiologic studies. assessment of the validity and reproducibility of a portable monitoring device. Chest. 1991; 100:1281-1286. [PubMed: 1935282]

20. Shaw JE, Punjabi NM, Wilding JP, Alberti KGMM, Zimmet PZ. Sleep-disordered breathing and type 2 diabetes: A report from the International Diabetes Federation Taskforce on Epidemiology and Prevention. Diabetes Research and Clinical Practice. 2008; 81:2-12. [PubMed: 18544448]

21. Al-Delaimy WK, Manson JE, Willett WC, Stampfer MJ, Hu FB. Snoring as a Risk Factor for Type II Diabetes Mellitus: A Prospective Study. American Journal of Epidemiology. 2002; 155:387393. [PubMed: 11867347]

22. Elmasry A, Janson C, Lindberg E, Gislason T, Tageldin MA, Boman G. The role of habitual snoring and obesity in the development of diabetes: a 10-year follow-up study in a male population. Journal of Internal Medicine. 2000; 248:13-20. [PubMed: 10947876]

23. Polak J, Shimoda LA, Drager LF, Undem C, McHugh H, Polotsky VY, et al. Intermittent hypoxia impairs glucose homeostasis in C57BL6/J mice: partial improvement with cessation of the exposure. Sleep. 2013; 36:1483-1490. 90a-90b. [PubMed: 24082307]

24. Somers VK, White DP, Amin R, Abraham WT, Costa F, Culebras A, et al. Sleep apnea and cardiovascular disease: an American Heart Association/american College Of Cardiology Foundation Scientific Statement from the American Heart Association Council for High Blood Pressure Research Professional Education Committee, Council on Clinical Cardiology, Stroke Council, and Council On Cardiovascular Nursing. In collaboration with the National Heart, Lung, and Blood Institute National Center on Sleep Disorders Research (National Institutes of Health). Circulation. 2008; 118:1080-1111. [PubMed: 18725495]

25. Henley DE, Russell GM, Douthwaite JA, Wood SA, Buchanan F, Gibson R, et al. HypothalamicPituitary-Adrenal Axis Activation in Obstructive Sleep Apnea: The Effect of Continuous Positive Airway Pressure Therapy. The Journal of Clinical Endocrinology \& Metabolism. 2009; 94:42344242. [PubMed: 19820009] 
26. Yamauchi M, Nakano H, Maekawa J, Okamoto Y, Ohnishi Y, Suzuki T, et al. Oxidative stress in obstructive sleep apnea. Chest. 2005; 127:1674-1679. [PubMed: 15888845]

27. Xie X, Pan L, Ren D, Du C, Guo Y. Effects of continuous positive airway pressure therapy on systemic inflammation in obstructive sleep apnea: A meta-analysis. Sleep Medicine. 2013; 14:1139-1150. [PubMed: 24054505]

28. Chin K, Shimizu K, Nakamura T, Narai N, Masuzaki H, Ogawa Y, et al. Changes in IntraAbdominal Visceral Fat and Serum Leptin Levels in Patients With Obstructive Sleep Apnea Syndrome Following Nasal Continuous Positive Airway Pressure Therapy. Circulation. 1999; 100:706-712. [PubMed: 10449691]

29. Schneider ALC, Pankow JS, Heiss G, Selvin E. Validity and Reliability of Self-reported Diabetes in the Atherosclerosis Risk in Communities Study. American Journal of Epidemiology. 2012; 176:738-743. [PubMed: 23013620] 


\section{Highlights}

- $\quad$ Obstructive sleep apnea (OSA) is a potentially modifiable risk factor for diabetes.

- We found that OSA was associated with greater risk of developing diabetes.

- $\quad$ The association between OSA and incident diabetes was independent of obesity.

- $\quad$ The relation persisted when the sample was restricted to individuals who were obese. 


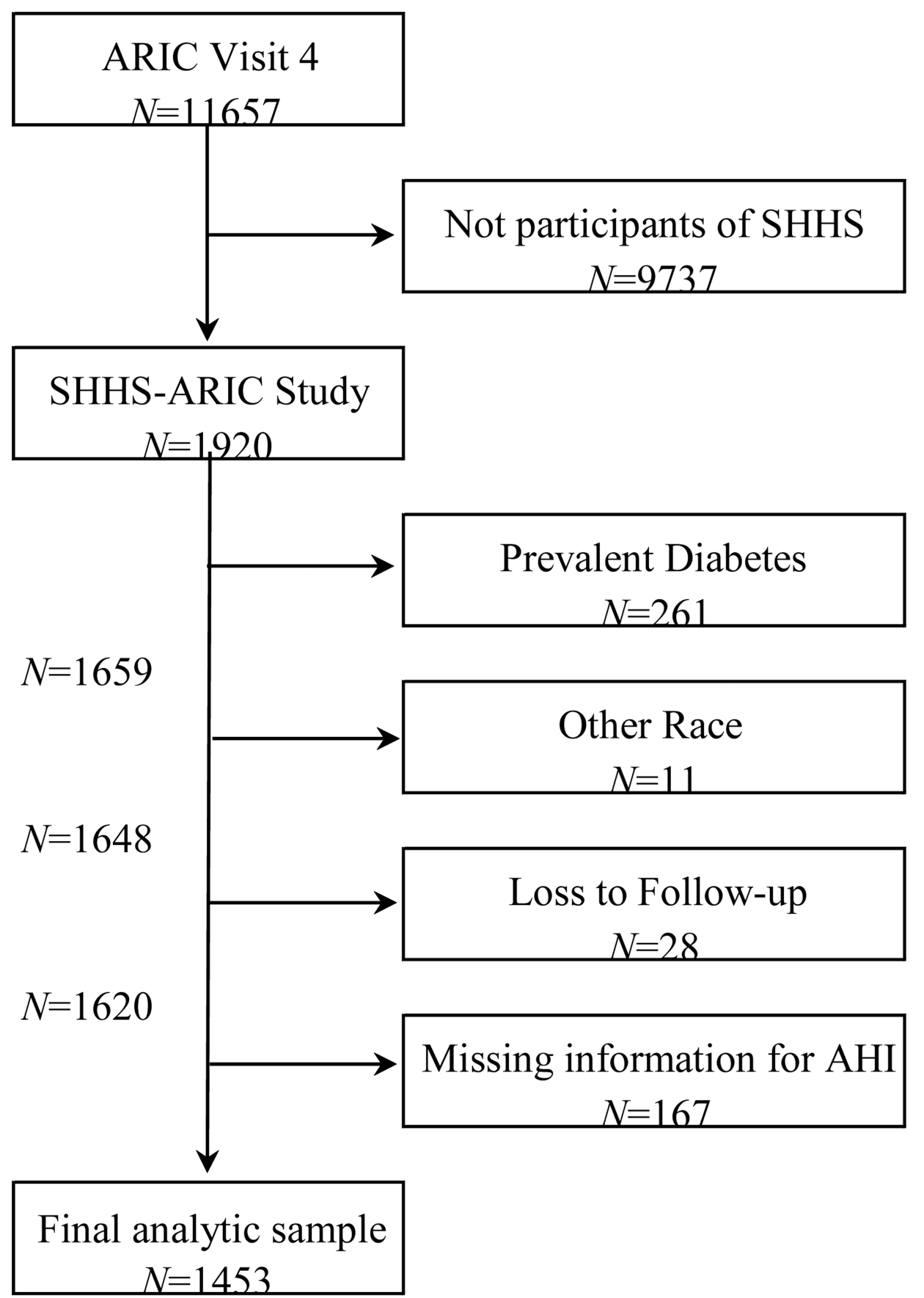

Fig. 1.

Study sample flow chart. AHI, apnea-hypopnea index, ARIC Study, Atherosclerosis Risk in Communities Study; SHHS, Sleep Heart Health Study. 


\section{을 \\ 올}

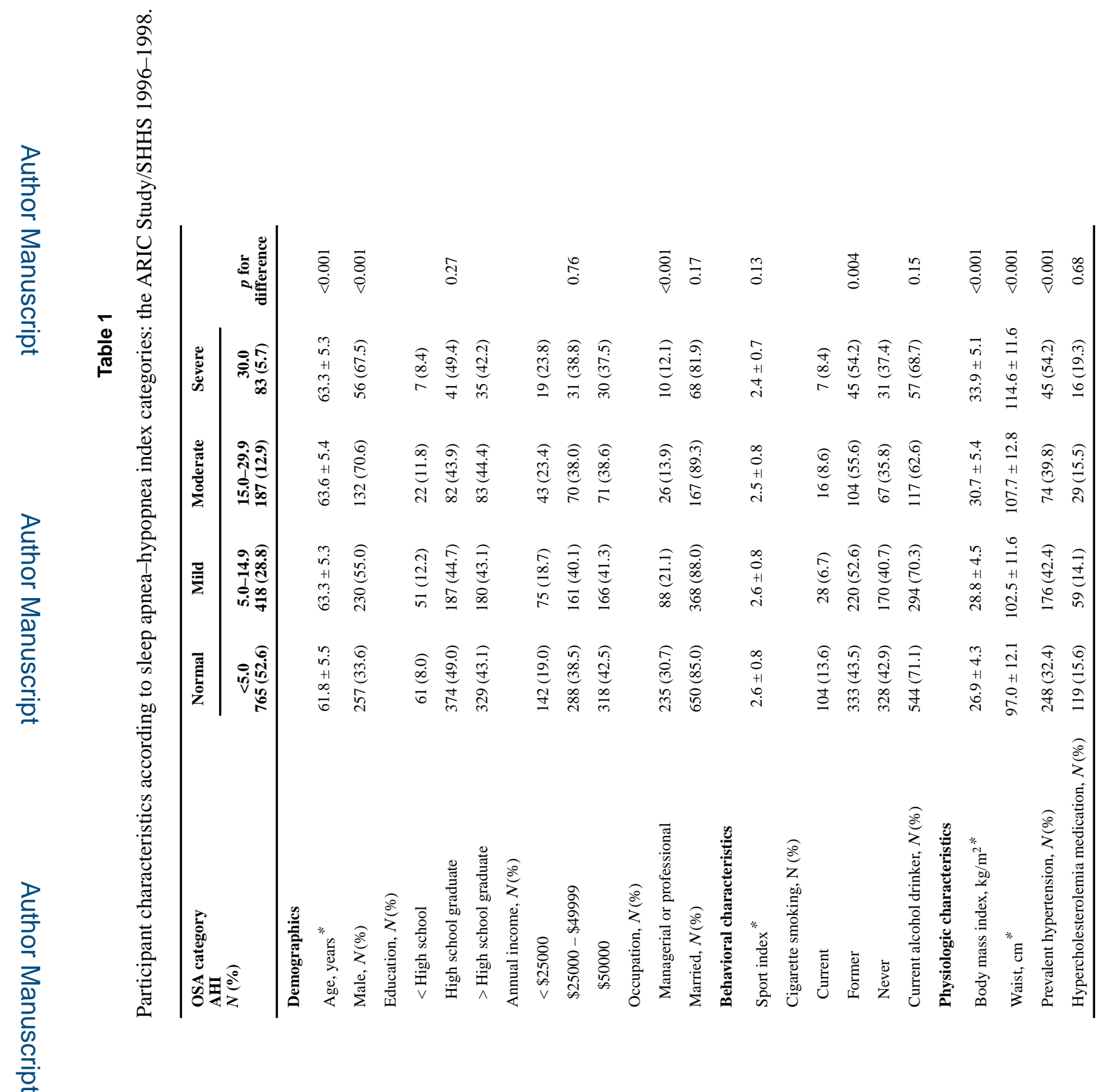

Sleep Med. Author manuscript; available in PMC 2017 September 29. 


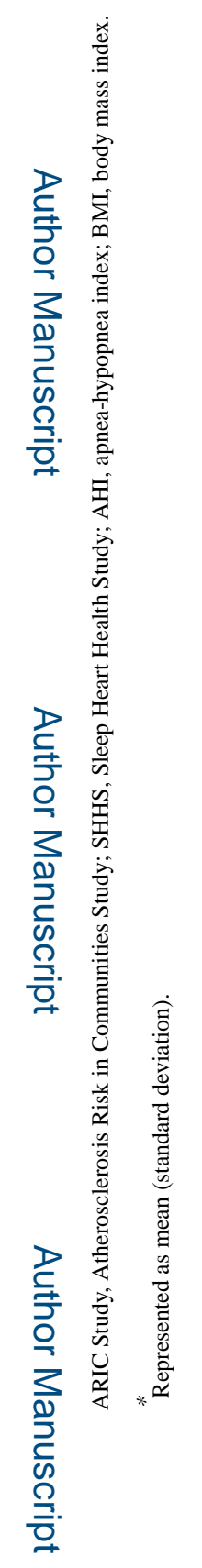

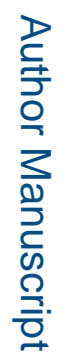

Sleep Med. Author manuscript; available in PMC 2017 September 29. 


\section{롤 \\ 길}

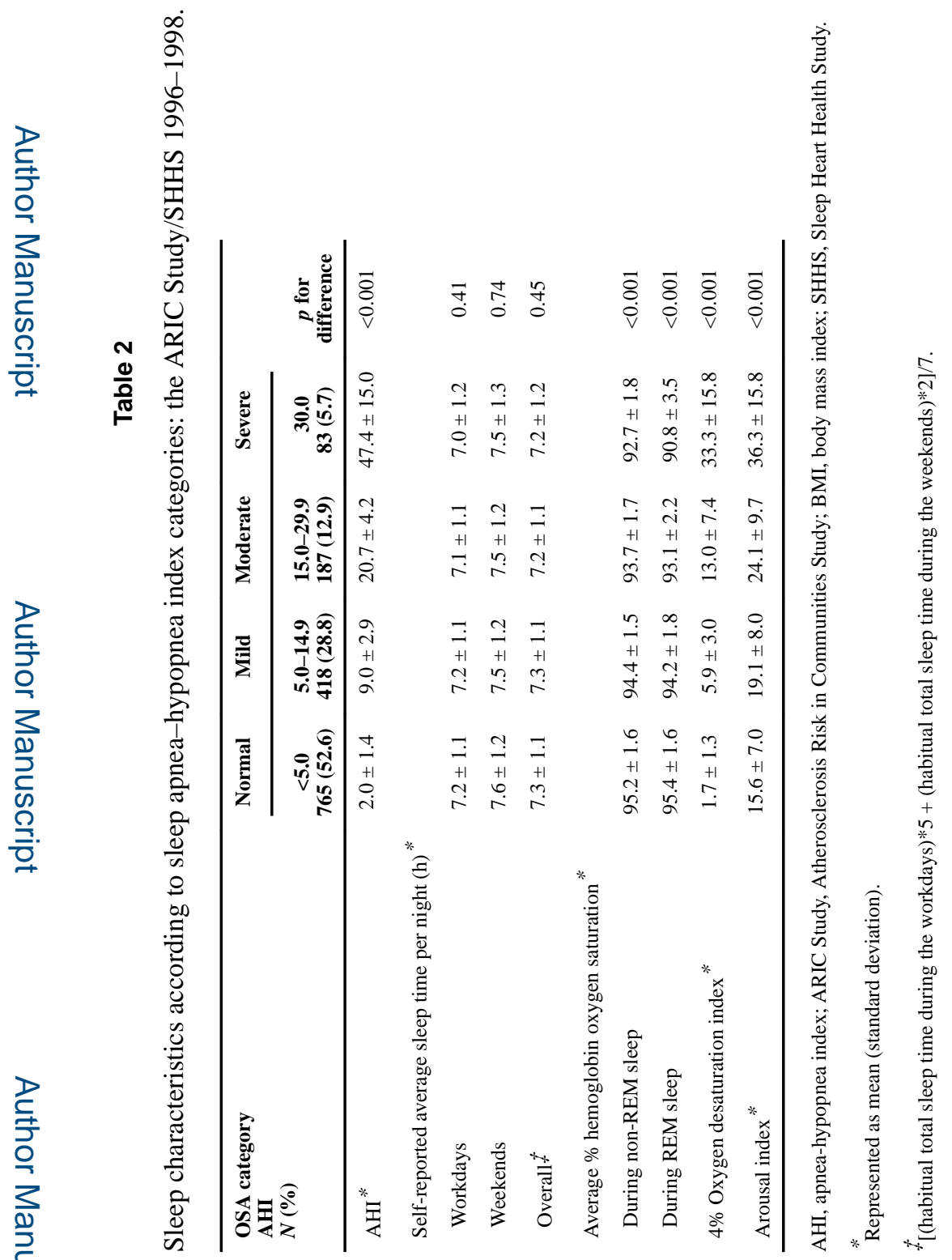




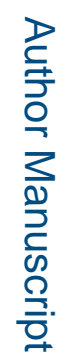

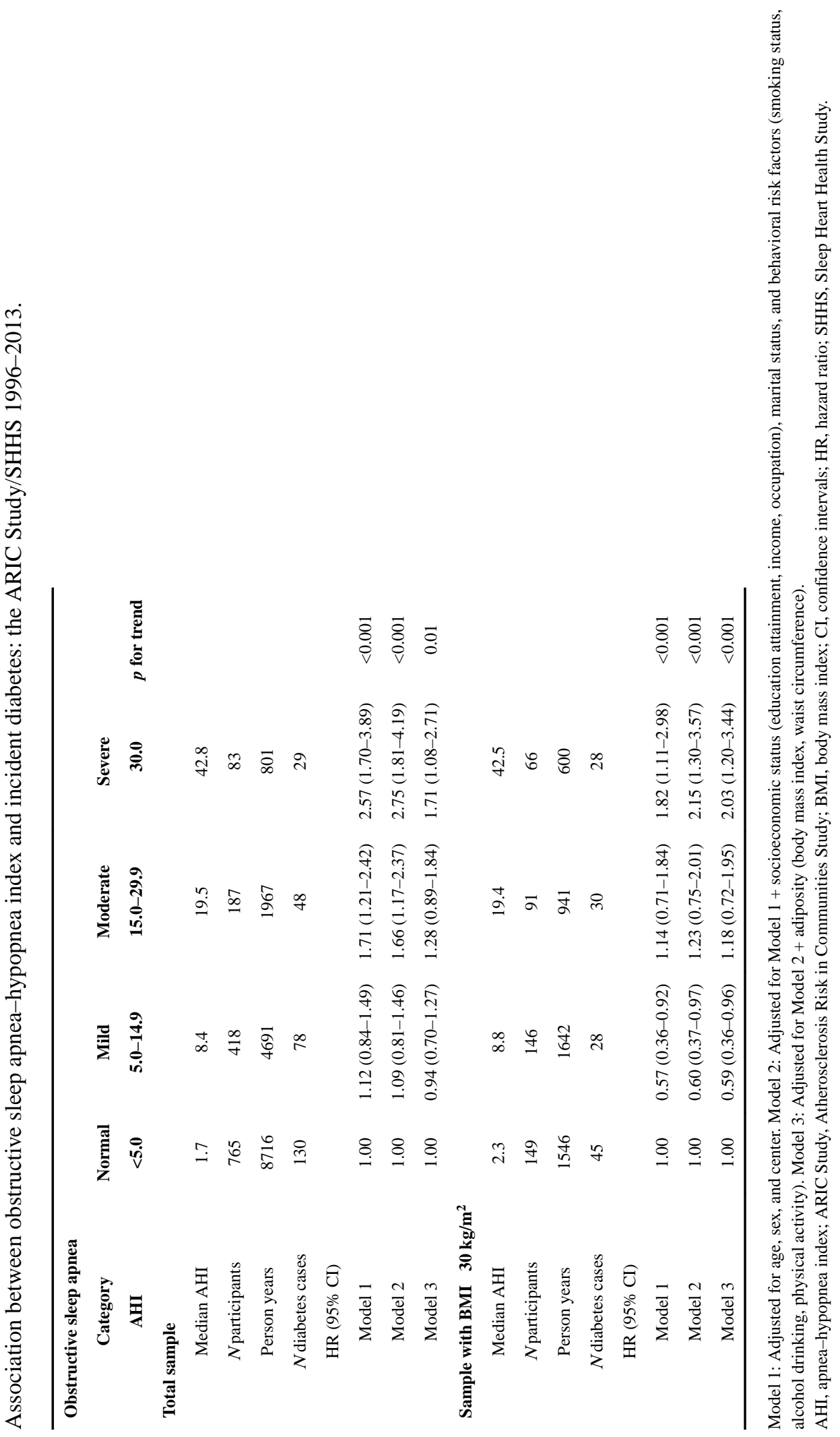

Sleep Med. Author manuscript; available in PMC 2017 September 29. 